\title{
Comparison of insulation provided by dry or wetsuits among recreational divers during cold water immersion $\left(<5^{\circ} \mathrm{C}\right)$
}

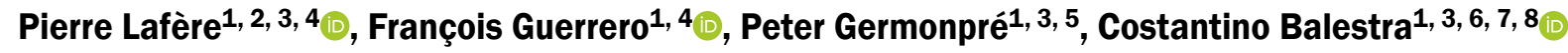 \\ ${ }^{1}$ DAN Europe Research Division \\ ${ }^{2}$ Department of Anaesthesiology, Erasme University Hospital, Université Libre de Bruxelles, Brussels, Belgium \\ ${ }^{3}$ Environmental, Occupational, Ageing (Integrative) Physiology Laboratory, Haute Ecole Bruxelles-Brabant (HE2B), Brussels, Belgium \\ ${ }^{4}$ Optimisation des Régulations Physiologiques (ORPHY), EA 4324, Université de Bretagne Occidentale, Brest, France \\ ${ }^{5}$ Centre for Hyperbaric Oxygen Therapy, Military Hospital "Queen Astrid", Brussels, Belgium \\ ${ }^{6}$ Anatomical Research and Clinical Studies (ARCS), Vrije Universiteit Brussel (V.U.B.), Brussels, Belgium \\ ${ }^{7}$ Anatomical Research Training and Education (ARTE), Vrije Universiteit Brussel (V.U.B.), Brussels, Belgium \\ ${ }^{8}$ Motor Sciences, Université Libre De Bruxelles (U.L.B.), Brussels, Belgium
}

\begin{abstract}
Background: Divers thermal status influences susceptibility to decompression sickness hence the need for proper insulation during immersion in cold water. However, there is a lack of data on thermal protection provided by diving suits, hence this study.

Materials and methods: Two different groups of divers wearing either a wetsuit $(n=15)$ or a dry suit $(n=15)$ volunteered for this study. Anthropometric data and dive experience were recorded; skin temperatures at the cervical-supraclavicular (C-SC) area and hands were assessed through high-resolution thermal infrared imaging taken pre- and post-dive.

Results: As far as anthropometrics, pre-dive C-SC temperatures $\left(37.0 \pm 0.4^{\circ} \mathrm{C}\right.$ ), depth (dry: $43 \pm 4.6 \mathrm{mfw}$ vs. wet: $40.3 \pm 4.0 \mathrm{mfw}$ ) and water temperature exposure $\left(4.3^{\circ} \mathrm{C}\right)$ are concerned, both groups were comparable. Total dive time was slightly longer for dry suit divers ( $39.6 \pm 4.0 \mathrm{~min}$ vs. $36.5 \pm 4.1 \mathrm{~min}, p=0.049$ ). Post-dive, C-SC temperature was increased in dry suit divers by $0.6 \pm 0.6^{\circ} \mathrm{C}$, and significantly decreased in wetsuit divers by $0.8 \pm 0.6^{\circ} \mathrm{C}$. The difference between groups was highly significant (dry: $37.5 \pm 0.7^{\circ} \mathrm{C}$ vs. wet: $36.2 \pm 0.7^{\circ} \mathrm{C}, p=0.004$ ). Hand's temperature decreased significantly in both groups (dry: $30.3 \pm$ $\pm 1.2^{\circ} \mathrm{C}$ vs. wet: $29.8 \pm 0.8^{\circ} \mathrm{C}, p=0.33$ ). Difference between groups was not significant.

Conclusions: Medium-duration immersion in cold water $\left(<5^{\circ} \mathrm{C}\right)$, of healthy and fully protected subjects was well tolerated. It was demonstrated that proper insulation based on a three-layer strategy allows maintaining or even slightly improve thermal balance. However, from an operational point of view, skin extremities are not preserved.
\end{abstract}

(Int Marit Health 2021; 72, 3: 217-222)

Key words: diving, cold water immersion, body insulation, wetsuits, dry suit, thermal imaging

\section{INTRODUCTION}

Although several studies have reported that moderate exercise in cold-water immersion, such as diving, facilitates overall heat loss because of increased convective heat transfer from the body core to the skin $[1,2]$, only scarce data are available on the thermoregulatory responses of fully protected divers exposed to cold water for prolonged time. Since divers need to avoid the "dive warm-decompress cool” pattern know to increase decompression risk substantially $[3,4]$, this might be considered a dangerous gap of

Pierre Lafère, PhD, Department of Anaesthesiology, Erasme University Hospital, Université Libre de Bruxelles, Brussels, Belgium, e-mail: pierre.lafere@erasme.ulb.ac.be 
knowledge. Indeed, recent studies point out an increased incidence of decompression sickness among finnish technical divers, whom performed long dives in areas where water temperature at the diving depths is $4-10^{\circ} \mathrm{C}$ even in summer [5], or among Belgian recreational divers when the winter is particularly cold [6].

Because most of the waters of the world are below thermoneutral $\left(34-35^{\circ} \mathrm{C}\right)$ temperature for humans, and there is no evidence that humans can increase their metabolism high enough to maintain core temperature during wholebody exposure to cold [7], insulating the body remains the only way to allow divers a prolonged stay under water [8]. Although the merit of different immersion suits has been extensively discussed as lifesaving appliance certified by the International Maritime Organization to ensure survival in case of accidental immersion in harsh conditions [9], less is known about diving suits especially among recreational divers. According to an older study, "dry" garments provide better protection than "wet" garments independently of sea conditions, and tight-fitting "wet" garments provided better protection than loose-fitting "wet" garments in rough but not in calm seas [10].

Therefore, evaluating the performance of clothing under severe cold environments becomes of great significance for divers' safety, hence this field study designed to compare body temperature variation related to insulation provided by wet or dry suits among recreational divers during an immersion of medium duration in very cold water $\left(<5^{\circ} \mathrm{C}\right)$.

\section{MATERIALS AND METHODS}

This study was conducted in accordance with the Declaration of Helsinki [11] and was part of a series of non-invasive studies carried out by the Environmental, Occupational, Ageing (Integrative) Physiology Laboratory, Haute Ecole Bruxelles-Brabant (HE2B), Brussels, Belgium, approved by the Academic Bioethical Committee of Brussels (B200-2011-5).

After being informed of the purpose and experimental procedures of the study, 30 male divers, with a minimal certification according to European norm EN 14153-2 or ISO 24801-2 and at least 60 dives in the last 2 years, volunteered and signed a written informed consent. They were recruited from a large diver population after visual pre-selection of possible candidates, to obtain a low variation in participants' anthropometric profiles to reduce the potential between-participant effect of body mass on preto post-dive core temperature measurements. To ensure that only reasonably healthy participants were included in the study, all participants completed a medical disclaimer. Age, height, weight, and dive experience were recorded for further analysis. Body fat percentage (\%BF) was derived from body circumference measurements (made in $\mathrm{cm}$ ) according to the US Navy formula [\%BF $=86.010 \times \log 10$ (abdomen - neck) $-70.041 \times \log 10$ (height) +30.30 ] $[12,13]$. Once recruited, participants were instructed to avoid alcohol beverages, smoking, caffeine, large meals, ointments, cosmetics and showering for 4 hours before the assessment.

All measurements were performed on the same day and dive site (Rochefontaine, Belgium), a flooded quarry with a maximal depth of 52 metres of fresh water ( $\mathrm{mfw}$ ). Participants dived according to their own dive plan (depth-time-breathing mixture). No specific dive profiles were imposed but subjects were asked to swim slowly to maintain a low to moderate-intensity energy expenditure related to exercise.

Based on their own dive gear that needed to be in good condition (no wear and tear) to participate, divers were allocated to two different groups: wetsuit divers (wet, $n=15$ : full $7 \mathrm{~mm}$ thick neoprene wetsuit combined with a $1.5 \mathrm{~mm}$ rash-guard undergarment [long sleeve top and pants], hood, boots, and gloves) or dry suit divers (dry, $\mathrm{n}=15$ : trilaminate membrane dry suit with high density polyester/merino wool fleece undergarment [top, legging, and socks] with neoprene $5 \mathrm{~mm}$ gloves and hood). Depth, total dive time and water temperature as measured by diver's own personal dive computer were noted to compare the level of cold exposure of the two groups.

During the examinations, the conditions were kept as identical as possible using the same procedure and type of equipment [14]. Since the condition of the skin surface may influence emissivity values, the skin was dried before the post-dive measurement. To limit the influence of friction on blood flow participants from both groups had to wipe oneself with a towel. More, to ensure a thermal sensitivity lower than $0.3^{\circ} \mathrm{C}$, all measurements were done in a heated dressing room, with a room temperature above $21^{\circ} \mathrm{C}$. There were no fans, and the doors were kept closed during the testing, so air movement was minimal. Then subjects were placed in a seated relaxed and upright position with arms adducted, away from all heat-emitting objects. Pictures of hands and left cervical-supraclavicular region (C-SC) at a 1.0-m distance from an IR thermal imaging camera (FLIR i7, FLIR System Inc, Wilsonville, Oregon, USA, thermal resolution at $19,600(140 \times 140)$ pixels, thermal sensitivity $<0.1^{\circ} \mathrm{C}$ and noise equivalent temperature difference $($ NETD) $<100 \mathrm{mK}$ ) were obtained from two different time points: one before and one within 10 minutes after completing their dive.

The stored thermographic images were analysed with R\&D FLIR Software (FLIR System Inc, Wilsonville, Oregon, USA). During data collection, the software was set to control the temperature scale limits between $28^{\circ} \mathrm{C}$ to $40^{\circ} \mathrm{C}$, which is the typical range of human body temperature. To allow the accurate, efficient, and reproducible identification of the region of interest (ROIs) they were defined as follow [15]: 



Figure 1. Comparison between pre- and post-dive cervical-supraclavicular (C-SC) and hands temperature after 40 minutes of exposure in cold water $\left(4^{\circ} \mathrm{C}\right)$. Divers were equipped either with a dry suit (dry) or a wetsuit (wet); NS - not significant; ${ }^{*} \mathrm{p}<0.05 ; * \star p<0.01$; $* * * p<0.001$

for the C-SC region, the limits of the ROI was calculated as straight lines from the neck and shoulder apices to the central apex and the shoulder contour between the shoulder and neck apices. The hottest $10 \%$ of points within this ROI were identified, and the supraclavicular temperature was defined as the median temperature of the hotspot [16, 17]; hand's temperature was assessed along a straight line positioned at the mid-dorsal side of all fingers. Each line extended from the middle of the nailbed and to the interdigital web which gave 10 average individual finger-temperatures that were used to calculate a mean temperature of the hands for each time point [18].

\section{STATISTICAL ANALYSIS}

Statistical analysis was performed using GraphPad Prism (version 5.0f for Mac, GraphPad Software, San Diego, California, USA). Since all data passed the Kolmogorov-Smirnov test, allowing us to assume a Gaussian distribution, comparison between variables was carried out with paired t-test for intragroup comparison and unpaired t-test for intergroup comparison. Data are expressed as mean \pm \pm standard deviation (SD). Differences between groups were considered significant at $p<0.05$.

\section{RESULTS}

As far as age, weight, height, body mass index (BMI), $\% \mathrm{BF}$ or numbers of logged dives are concerned; both groups were comparable (Table 1). Cold exposure was also similar in terms of water temperature $(4.3 \pm 0.6$ vs. $4.3 \pm 0.7$, unpaired t-test, $p=0.835, \mathrm{df}=28$ ) and maximal depth ( $43 \pm 4.6$ vs. $40.3 \pm 4.0 \mathrm{mfw}$, unpaired t-test, $p=0.101$, $\mathrm{df}=28$ ). Nonetheless, there is a 3-minute difference in total dive time between groups ( $39.6 \pm 4.0 \mathrm{~min}$ vs. $36.5 \pm$
Table 1. Group's anthropometrics and dive experience (unpaired t-test, $\mathrm{df}=28$ )

\begin{tabular}{lcc}
\hline & Dry & Wet \\
\hline Age [years] & $36 \pm 6$ & $36 \pm 6$ \\
Weight [kg] & $77 \pm 8$ & $80 \pm 10$ \\
Height [cm] & $180 \pm 6$ & $181 \pm 6$ \\
Body mass index & $23.7 \pm 1.7$ & $24.4 \pm 1.8$ \\
Body fat percentage & $18.9 \pm 6.3$ & $18.7 \pm 5.5$ \\
Logged dives $(n)$ & $595 \pm 218$ & $458 \pm 247$
\end{tabular}

$\pm 4.1 \mathrm{~min}$, unpaired t test, $\mathrm{p}=0.049$, $\mathrm{df}=28$ ). Although just statistically significant, this might be not practically relevant.

Pre-dive C-SC (dry: $36.9 \pm 0.5^{\circ} \mathrm{C}$ vs. wet: $37.0 \pm 0.4^{\circ} \mathrm{C}$, unpaired t-test, $p=0.39, \mathrm{df}=28$ ) and hands (dry: $34.7 \pm$ $\pm 0.6^{\circ} \mathrm{C}$ vs. wet: $34.7 \pm 0.4^{\circ} \mathrm{C}$, unpaired t-test, $p=0.97$, $\mathrm{df}=28$ ) temperatures were similar in both groups.

Variations of C-SC and hands temperatures are described in Figure 1. Immediately after the dive, C-SC temperature was increased by $0.6 \pm 0.6^{\circ} \mathrm{C}$ in dry suit divers to $37.5 \pm 0.7^{\circ} \mathrm{C}$ (paired t-test, $p=0.004$, df $=14$ ), while it was significantly decreased by $0.8 \pm 0.6^{\circ} \mathrm{C}$ in wetsuit divers to $36.2 \pm 0.7^{\circ} \mathrm{C}$ (paired t-test, $p=0.0001, d f=14$ ). The post-dive C-SC temperature difference between groups was highly significant (unpaired t-test, $p<0.0001, d f=28$ ). Hand's temperature decreased significantly in both groups (dry: $30.3 \pm 1.2^{\circ} \mathrm{C}$ vs. wet: $29.8 \pm 0.8^{\circ} \mathrm{C}$, paired t-test, $p<0.0001, d f=14)$. Although dry suit divers had slightly warmer hands than wetsuit divers after the dive, this difference between groups was not significant (unpaired t-test, $p=0.33, d f=28$. 


\section{DISCUSSION}

Evaluating the performance of clothing under severe thermal environments is not an easy task, especially in the field. The most appropriate method for measuring the thermal resistance provided by the garments would be to measure heat flow across the insulation layer. That would typically be done in a laboratory setup using the most common tool: the thermal manikin [19]. However, it is associated with significant discrepancies between results obtained from the three proposed calculation methods either global, serial, or parallel, which are particularly relevant with cold exposure [20], as in the present setting. Also, from the physiological standpoint, this might not be adapted as we are more interested on how the divers' thermal balance to cold exposure is modified by any type of diving suit rather than the insulation perse. Then, alternative means would be to measure skin temperature at multiple sites as body heat loss will depend on the heat flux from different sites [21]. Although, these thermocouples are non-invasive, the required hard-wired connections to a datalogger limit its validity and fail to address questions and applications outside the laboratory. Therefore, we opted for infra-red thermal imaging as a surrogate method for body temperature monitoring.

Although this technique has been shown to be valid and reliable [22], and recommended for clinical use in inflammatory diseases, complex regional pain syndrome, Raynaud's phenomenon, as well as for fever screening [23], some limitations must be acknowledged. First, large errors versus a thermocouple during rest and exercise have been reported, making it unclear within which situations it may have application. One study whose aims was to investigate the validity and reliability of skin temperature measurements using different portable system against a certified thermocouple, demonstrated that thermal cameras did not perform as well as the other devices when providing live, handheld measurements of skin temperature during exercise. However, it also demonstrated that thermal cameras are useful tools for measuring skin temperature in static and controlled environments [24], as in our study. Second, the principle of thermal cameras is based on emissivity, namely the ability of the skin to emit energy by radiation, which is processed to calculate the temperature of the studied surface. Although, this method limits inter-individual variation and the consequential non-uniform heat production [24], heat transfer and temperature gradients from body core to skin surface is under the influence of many factor such as skin wettedness, ambient temperature once disrobed, duration of exposure to the ambient temperature or subcutaneous fat and skin. Most of these parameters were accounted for. For instance, there are no gender, ethnic, age or BMI differences in our population. It can be considered that the selected method to estimate
$\% \mathrm{BF}$, even if not perfectly accurate, provides sufficiently valid results also demonstrating no difference between our two groups. Therefore, there is a reasonable assumption to neglect any in-between group's bias related to fat insulation or measurements conditions. Indeed, a compromise must be made between increasing measurement accuracy and maintaining validity of the protocol, when trying to transfer otherwise valid tools to applied situations.

Our results are consistent with previous observations, demonstrating that wetsuit diving in cold-water results in a drop-in body temperature and may not provide adequate thermal protection in such condition $[25,26]$. The next expected observation should have been a slowing down of heat loss with a limited drop of skin temperature while wearing a dry suit compared to a wetsuit. Surprisingly, we observed the opposite, namely an increased C-SC skin temperature among dry suit divers.

Two hypotheses may explain these results. First, cold immersion is known to increase heat production as a function of both core/skin temperature and exercise (in our case, finning) [27]. This production in combination with fabrics with a higher thermal insulation such as fleece used in the present setting, would allow a higher temperature than neoprene or other membrane fabric. Although one could argue that human-clothing-environment simulator is less relevant than the use of cold water due to the thermal properties of water, similar results have been demonstrated for outdoor undergarments [28]. Second, the reduction of C-SC skin temperature among wetsuit divers could be due to vasoconstricted skin on the chest, which would result in cooler skin temperatures. Therefore, the gradient between core and skin may not have been established and it is not certain that deep body cooling occurs in wet suited divers. However, based on previous literature [25, 26], this hypothesis is unlikely and does not explain the results observed among dry suit divers. Indeed, this has recently been demonstrated among spinal cord injured divers wearing a wetsuit while exposed to $6^{\circ} \mathrm{C}$ water dive. Although the drop was variable, the decrease in core temperature was significantly increased in the absence of thermoregulatory control below the lesion compared to able-bodied diver [29].

Based on the starting and ending cylinder pressures, equivalent surface air consumption was calculated around $17.5 \mathrm{~L} / \mathrm{min}$ in both groups. Exercise intensity inferred from these estimates corresponds to a moderate-intensity energy expenditure (5.3 to 5.8 MET) [30]. Since both groups showed similar exposure, the difference in C-SC skin temperature between groups is likely to be due to the suit worn. Because of a positive association between surface and core body [31], it seems a reasonable assumption to consider that C-SC skin temperature reflects core body temperature patterns, but not necessarily absolute core temperature [32]. Then, as 
a conclusion, compared to wetsuits, it would be logical to assume that dry suit better preserve core temperature as this condition is associated with both an increase of C-SC skin temperature and an alleged increased core-to-skin temperature gradient.

It is also known that cold exposure is associated with hand dysfunction unless body heat content is maintained [10]. Therefore, it is paramount to preserve both core and hand's temperature. This can be achieved either by insulation or by using some heating device, but both seem to be ineffective. Indeed, according to previous literature, neither manual dexterity nor finger temperature could be rescued using whole body active heating [21], while in wet-suited subjects, wearing gloves and boots seems to increase the overall rate of body heat loss [8, 33]. Consistently with these findings, no difference in hand's temperature among groups was observed and pain in the hands with major discomfort was the main complaint in both groups at the end of the dive. Although hypothetical, similar hand's temperature could paradoxically be explained by a better preservation of core temperature among dry suit divers associated with a reduction of cold-induced vasoconstriction. This mechanism has been proposed after an experiment comparing two forms of hand heating, either direct hand heating with electrically heated gloves or indirectly by actively heating the torso with an electrically heated vest, while exposed to minus $25^{\circ} \mathrm{C}$ in air. Despite a similar finger skin temperature, the finger blood flow was significantly higher during the heated vest condition [34]. Indeed, compared to the C-SC skin temperature, which is thought to reflect core body temperature patterns, we observed a greater magnitude of the changes in the dry suit condition (dry: $-7.2 \pm 1.2^{\circ} \mathrm{C}$ vs. wet: $-6.3 \pm 0.8^{\circ} \mathrm{C}$ ). Consequently, comfort and dexterity may be the limiting factor in performance/endurance irrespective of how well the rest of the body is protected. It is indeed a known fact that manual gross and fine dexterity decreases by $71.6 \pm$ $\pm 22.9 \%$ and $44.5 \%$, respectively during cold water $\left(10^{\circ} \mathrm{C}\right) \mathrm{im}$ mersion and continue to decrease with immersion time [21].

\section{CONCLUSIONS}

In conclusion, medium-duration immersion in cold water less than $5^{\circ} \mathrm{C}$, of healthy and fully protected subjects was well tolerated. It was demonstrated that insulation based on a three-layer strategy (a base layer to wick water away from the skin to reduce conductive heat loss to the liquid; a mid-layer to provides insulation; and the dry suit membrane to provide a barrier to convective heat loss) might maintain or even slightly increase body temperature. However, cooling of the hands, with impairment of dexterity in such conditions, may constitute a major safety issue.

\section{Conflict of interest: None declared}

\section{REFERENCES}

1. Ferretti $G$, Veicsteinas $A$, Rennie DW. Regional heat flows of resting and exercising men immersed in cool water. J Appl Physiol (1985). 1988; 64(3): 1239-1248, doi: 10.1152/jappl.1988.64.3.1239, indexed in Pubmed: 3366739.

2. McArdle WD, Toner MM, Magel JR, et al. Thermal responses of men and women during cold-water immersion: influence of exercise intensity. Eur J Appl Physiol Occup Physiol. 1992; 65(3): 265-270, doi: 10.1007/BF00705092, indexed in Pubmed: 1396657.

3. Gerth W, Ruterbusch V, Long E. The influence of thermal exposure on diver susceptibility to decompression sickness. Panama City: Navy Experimental Diving Unit, 2007 Contract No.: NEDU TR 06-07. 2007, doi: 10.21236/ada480118.

4. Pollock NW, editor Thermal physiology and diver protection. Rebreather Forum 3; 2013; Durham, NC: AAUS/DAN/PADI.

5. Lundell RV, Arola O, Suvilehto J, et al. Decompression illness (DCl) in Finland 1999-2018: Special emphasis on technical diving. Diving Hyperb Med. 2019; 49(4): 259-265, doi: 10.28920/dhm49.4.259265, indexed in Pubmed: 31828744.

6. Lafère P, Balestra C, Caers D, et al. Patent Foramen Ovale (PFO), Personality Traits, and Iterative Decompression Sickness. Retrospective Analysis of 209 Cases. Front Psychol. 2017; 8: 1328, doi: 10.3389/ fpsyg.2017.01328, indexed in Pubmed: 28824507.

7. Senn JR, Maushart $\mathrm{Cl}$, Gashi $\mathrm{G}$, et al. Outdoor temperature influences cold induced thermogenesis in humans. Front Physiol. 2018; 9: 1184, doi: 10.3389/fphys.2018.01184, indexed in Pubmed: 30190681.

8. Park YS, Kim JS, Choi JK. Increase of heat loss by wearing gloves and boots in wet-suited subjects working in cold water. Ann Physiol Anthropol. 1992; 11(4): 393-400, doi: 10.2114/ahs1983.11.393, indexed in Pubmed: 1388402.

9. Power J, Tikuisis P, Ré AS, et al. Correction factors for assessing immersion suits under harsh conditions. Appl Ergon. 2016; 53 Pt A: 87-94, doi: 10.1016/j.apergo.2015.08.009, indexed in Pubmed: 26674408.

10. Steinman AM, Hayward JS, Nemiroff MJ, et al. Immersion hypothermia: comparative protection of anti-exposure garments in calm versus rough seas. Aviat Space Environ Med. 1987; 58(6):550-558, indexed in Pubmed: 3606516.

11. World Medical Association. World Medical Association Declaration of Helsinki: ethical principles for medical research involving human subjects. JAMA. 2013; 310(20): 2191-2194, doi: 10.1001/ jama.2013.281053, indexed in Pubmed: 24141714.

12. Hodgdon JA, Beckett MB. Prediction of percent body fat for U.S. Navy men from body circumferences and height. 1984, doi: 10.21236/ ada143890.

13. Hodgdon J, Friedl K. Development of the DoD Body Composition Estimation Equations. San Diego, CA: US Navy, 1998 Technical Document No. 99-2B. 1998, doi: 10.21236/ada370158.

14. Moreira DG, Costello JT, Brito CJ, et al. Thermographic imaging in sports and exercise medicine: A Delphi study and consensus statement on the measurement of human skin temperature. J Therm Biol. 2017; 69: 155-162, doi: 10.1016/j.jtherbio.2017.07.006, indexed in Pubmed: 29037377.

15. Tipton MJ, Collier N, Massey H, et al. Cold water immersion: kill or cure? Exp Physiol. 2017; 102(11): 1335-1355, doi: 10.1113/ EP086283, indexed in Pubmed: 28833689.

16. Larsen T, Kumar S, Grimmer K, et al. A systematic review of guidelines for the prevention of heat illness in community-based sports participants and officials. J Sci Med Sport. 2007; 10(1): 11-26, doi: 10.1016/j.jsams.2006.07.008, indexed in Pubmed: 16952483. 
17. Law J, Morris DE, Izzi-Engbeaya C, et al. Thermal imaging is a noninvasive alternative to PET/CT for measurement of brown adipose tissue activity in humans. J Nucl Med. 2018; 59(3): 516-522, doi: 10.2967/jnumed.117.190546, indexed in Pubmed: 28912148.

18. Norheim AJ, Borud E, Wilsgaard T, et al. Variability in peripheral rewarming after cold stress among 255 healthy Norwegian army conscripts assessed by dynamic infrared thermography. Int J Circumpolar Health. 2018; 77(1): 1536250, doi: 10.1080/22423982.2018.1536250, indexed in Pubmed: 30444464.

19. Ergonomics of the thermal environment. Estimation of thermal insulation and water vapour resistance of a clothing ensemble. ISO 9920. 2010, doi: $10.3403 / 30125323$.

20. Oliveira AV, Gaspar AR, Quintela DA. Measurements of clothing insulation with a thermal manikin operating under the thermal comfort regulation mode: comparative analysis of the calculation methods. Eur J Appl Physiol. 2008; 104(4): 679-688, doi: 10.1007/s00421008-0824-5, indexed in Pubmed: 18633635.

21. Wheelock CE, Hess HW, Schlader ZJ, et al. Whole-body active heating does not preserve finger temperature or manual dexterity during cold-water immersion. Undersea Hyperb Med. 2020; 47(2): 253-260, indexed in Pubmed: 32574442.

22. Roy R, Boucher JP, Comtois AS. Validity of infrared thermal measurements of segmental paraspinal skin surface temperature. J Manipulative Physiol Ther. 2006; 29(2): 150-155, doi: 10.1016/j. jmpt.2005.12.004, indexed in Pubmed: 16461175.

23. Ring EFJ, Ammer K. Infrared thermal imaging in medicine. Physiol Meas. 2012; 33(3): R33-R46, doi: 10.1088/0967-3334/33/3/ R33, indexed in Pubmed: 22370242.

24. James CA, Richardson AJ, Watt PW, et al. Reliability and validity of skin temperature measurement by telemetry thermistors and a thermal camera during exercise in the heat. J Therm Biol. 2014; 45: 141-149, doi: 10.1016/j.jtherbio.2014.08.010, indexed in Pubmed: 25436963.

25. Arieli R, Kerem D, Gonen A, et al. Thermal status of wet-suited divers using closed circuit 02 apparatus in sea water of 17-18.5 degrees C.
Eur J Appl Physiol Occup Physiol. 1997; 76(1): 69-74, doi: 10.1007/ s004210050214, indexed in Pubmed: 9243172.

26. Shiraki K, Sagawa S, Konda N, et al. Energetics of wet-suit diving in Japanese male breath-hold divers. J Appl Physiol (1985). 1986; 61(4): 1475-1480, doi: 10.1152/jappl.1986.61.4.1475, indexed in Pubmed: 3781961.

27. Parsons KC. Human Thermal Environments. The effects of hot, moderate and cold environments on human health, comfort and performance. . 2nd ed. Taylor \& Francis, London 2003.

28. Kim E, Yoo SJ, Shim H. Performance of selected clothing systems under subzero conditions: determination of performance by a human-clothing-environment simulator. Text Res J. 2016; 76(4): 301-308, doi: 10.1177/0040517506057529.

29. Gajsek U, Sieber A, Finderle Z. Thermal balance of spinal cord injured divers during cold water diving: a case control study. Diving Hyperb Med. 2020; 50(3): 256-263, doi: 10.28920/dhm50.3.256-263, indexed in Pubmed: 32957128.

30. Buzzacott P, Pollock NW, Rosenberg M. Exercise intensity inferred from air consumption during recreational scuba diving. Diving Hyperb Med. 2014; 44(2): 74-78, indexed in Pubmed: 24986724.

31. McFarland R, Barrett L, Fuller A, et al. Infrared thermography cannot be used to approximate core body temperature in wild primates. Am J Primatol. 2020; 82(12): e23204, doi: 10.1002/ajp.23204, indexed in Pubmed: 33043502.

32. van der Vinne V, Pothecary CA, Wilcox SL, et al. Continuous and non-invasive thermography of mouse skin accurately describes core body temperature patterns, but not absolute core temperature. Sci Rep. 2020; 10(1): 20680, doi: 10.1038/s41598-020-77786-5, indexed in Pubmed: 33244132.

33. Riera F, Horr R, Xu X, et al. Thermal and metabolic responses of military divers during a 6-hour static dive in cold water. Aviat Space Environ Med. 2014; 85(5): 509-517, doi: 10.3357/asem.3077.2014, indexed in Pubmed: 24834564.

34. Brajkovic D, Ducharme MB. Finger dexterity, skin temperature, and blood flow during auxiliary heating in the cold. J Appl Physiol (1985). 2003; 95(2): 758-770, doi: 10.1152/japplphysiol.00051.2003, indexed in Pubmed: 12730145. 